Clinical Image

Robert Dazé*, DO, Jorge Canales, DO and Risa Ross, DO, FAOCD

\title{
Kaposi varicelliform eruption in a patient with atopic dermatitis
}

https://doi.org/10.1515/jom-2021-0170

Received June 19, 2021; accepted June 25, 2021;

published online July 15, 2021

A 23 year old woman with a history of uncontrolled atopic dermatitis presented to the emergency department with an acute onset of a painful papulovesicular facial rash that developed 1 week prior to presentation in September 2020.

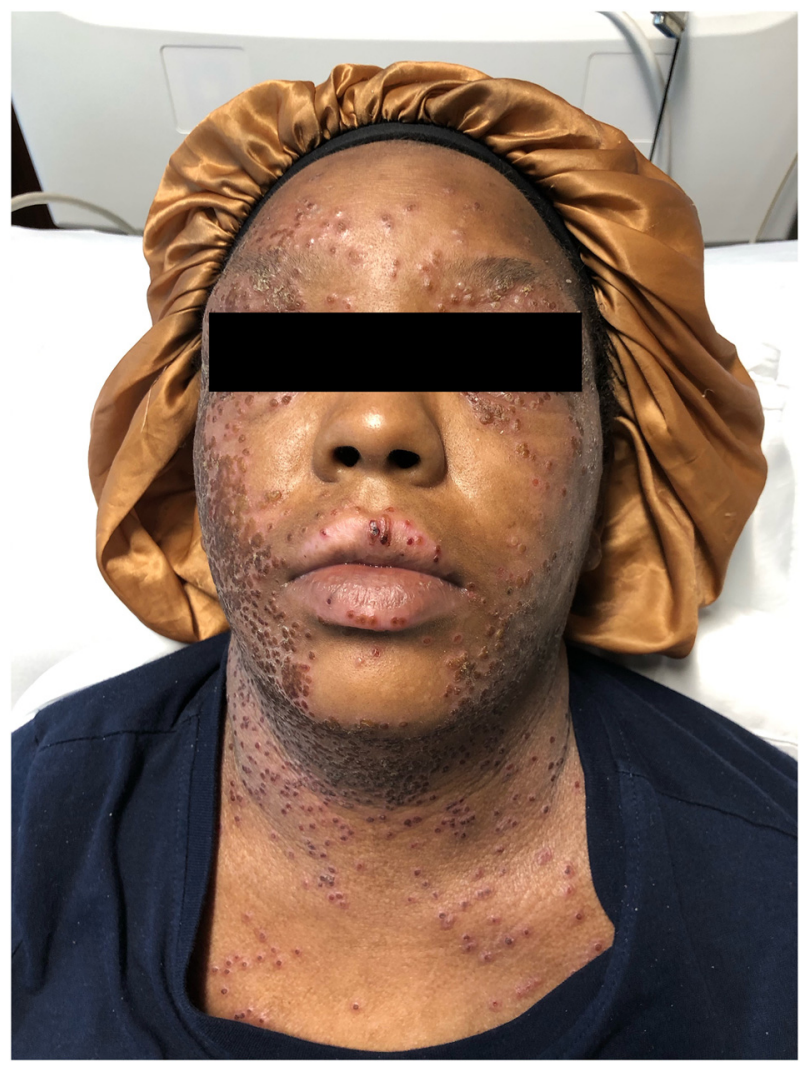

Image A

*Corresponding author: Robert Dazé, DO, Dermatology Resident, HCA Healthcare/USF Morsani College of Medicine, Largo Medical Center in Largo, 871 lbis Walk Place North, Unit 8108, St. Petersburg, FL 33716, USA, E-mail: rdaze022@marian.edu

Jorge Canales, DO and Risa Ross, DO, FAOCD, Internal Medicine Resident, Board-Certified Dermatologist, HCA Healthcare/USF Morsani College of Medicine, Largo Medical Center in Largo, St. Petersburg, FL, USA

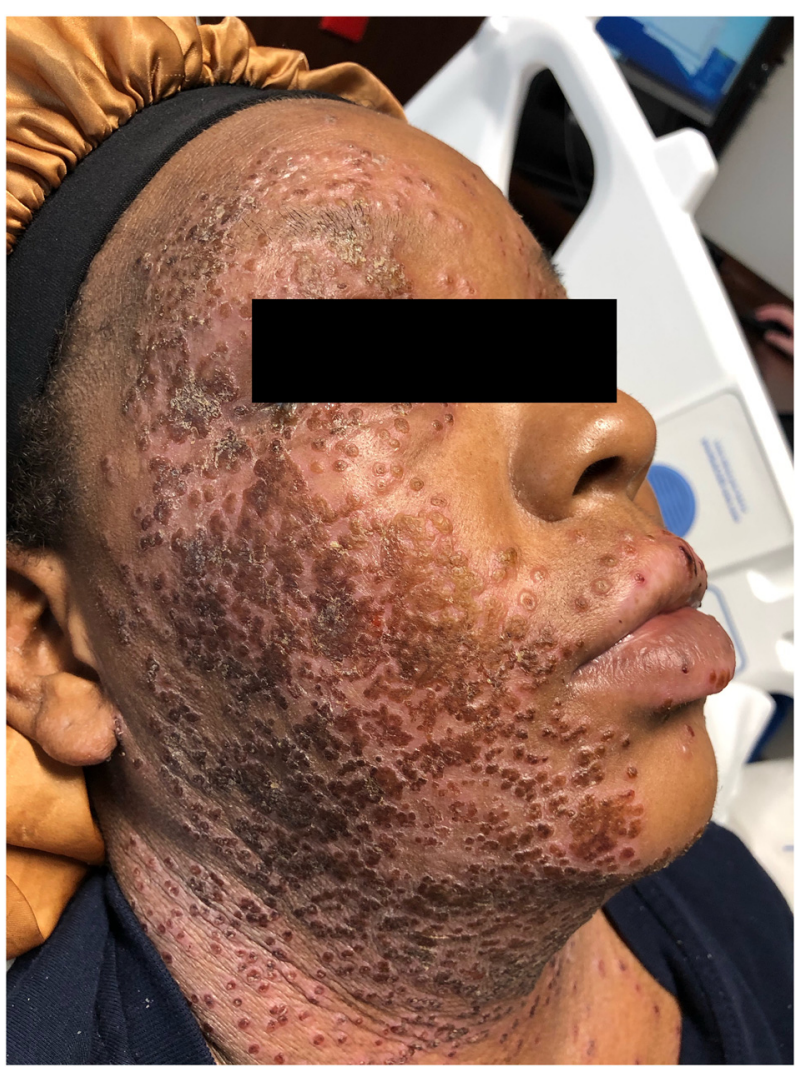

Image B

Review of systems was positive for crusting and swelling of both eyes, bilateral cervical lymphadenopathy, and headache. Physical exam demonstrated multiple umbilicated vesicles and pustules with serous crusting (Images A and B). Bilateral ocular exudate was noted without any conjunctival injection. Herpes simplex virus 1 (HSV-1) DNA was detected by polymerase chain reaction. The patient was diagnosed with Kaposi varicelliform eruption and successfully treated with acyclovir.

Kaposi varicelliform eruption, also known as eczema herpeticum, is a cutaneous dissemination of a viral etiology in the setting of certain underlying skin diseases, most commonly atopic dermatitis [1]. This is usually due to HSV-1 but can be complicated by a bacterial superinfection such as Staphylococcus aureus or group A Streptococcus [2]. It 
mostly affects infants and children, but it can present in any age demographic. Individuals present with a monomorphic eruption of umbilicated papules and vesicopustules and associated systemic symptoms such as fever, malaise, and lymphadenopathy [1-3]. Diagnosis can be confirmed with viral cultures, direct fluorescent antibody assays, polymerase chain reaction, and serologic studies [2]. Serologic testing has a limited diagnostic capacity notably in seroconverted patients, as it will not be able to distinguish if the active infection is due to this virus or another etiology [2]. Ocular sequelae including herpes keratitis is a serious complication and should be urgently evaluated by ophthalmology. In infants and young children, this condition is considered a medical emergency as systemic viremia can result in significant morbidity and mortality [2]. The mainstay treatment is with a nucleoside analog antiviral such as acyclovir, valacyclovir, or famciclovir [3]. Intravenous delivery should be considered in neonates, immunosuppressed patients, and those with severe or systemic complications. Antibiotics are not indicated unless there is any secondary bacterial impetiginization.

Research funding: None reported.

Author contributions: All authors provided substantial contributions to conception and design, acquisition of data, or analysis and interpretation of data; all authors drafted the article or revised it critically for important intellectual content; all authors gave final approval of the version of the article to be published; and all authors agree to be accountable for all aspects of the work in ensuring that questions related to the accuracy or integrity of any part of the work are appropriately investigated and resolved.

Competing interests: None reported.

Informed consent: The patient in this report provided written informed consent.

\section{References}

1. Wollenberg A, Zoch C, Wetzel S, Plewig G, Przybillaa B. Predisposing factors and clinical features of eczema herpeticum: a retrospective analysis of 100 cases. J Am Acad Dermatol 2003;49: 198-205.

2. Downing C, Mendoza N, Sra K, Tyring SK. Human herpesviruses. In: Bolongia JL, Schaffer JV, Cerroni L, eds. Dermatology, 4th ed. Philadelphia: Elsevier; 2018:1403-6 pp.

3. Kramer SC, Thomas CJ, Tyler WB, Elston DM. Kaposi's varicelliform eruption: a case report and review of the literature. Cutis 2004;73: 115-22. 